\title{
DISTRIBUTION OF INTEGERS WITH PRESCRIBED STRUCTURE AND APPLICATIONS
}

\author{
KAM HUNG YAUD \\ (Received 12 August 2020; first published online 19 October 2020)
}

2020 Mathematics subject classification: primary 11N25; secondary 11J20, 11L05, 11L40, 11N36, 11P32. Keywords and phrases: character sum, Harman sieve, Kloostermann sum, local model, smooth numbers.

In this thesis, we are concerned with the distribution of integers with prescribed arithmetic structure. We also include an application to bounds of character sums in Chapter 6.

In Chapter 1 , we consider a problem in Diophantine approximation. Let $\|\cdot\|$ be the distance to the nearest integer function. For any $\varepsilon>0$, we acquire an asymptotic formula for the number of solutions $n \leq x$ to

$$
\|\alpha n+\beta\|<x^{-1 / 4+\varepsilon},
$$

where $n$ is $[y, z]$-smooth (numbers whose prime factors lie in the interval $[y, z] \subseteq[1, x]$ ) for infinitely many real numbers $x$. In addition, we also establish an asymptotic formula with an extra square-free condition on $n$. Moreover, if $\alpha$ is quadratic irrational, then the asymptotic formulas hold for all sufficiently large $x$.

Our tools come from the Harman sieve [6], which we adapt suitably to sieve for $[y, z]$-smooth numbers. The arithmetic information comes from estimates for exponential sums. The results in Chapter 1 have been published in [11].

In Chapter 2, we consider a variant of a conjecture (EOSC) due to Erdôs et al. [4], which states that for all sufficiently large $q$, every reduced residue class modulo $q$ can be written as a product of two primes, each no more than $q$. We establish estimates for the number of ways to represent any reduced residue class as a product of a prime and an integer free of small prime factors. That is, for $(a, q)=1$ and a positive real number $z$, we count the number of pairs $(p, u)$ such that

$$
p u \equiv a(\bmod q),
$$

where prime $p \leq x, u \leq y$ and the smallest prime factor of $u$ is no less than $z$. The best results we obtain are conditional on the generalised Riemann hypothesis (GRH). Our

Thesis submitted to the University of New South Wales in February 2020; degree approved on 10 June 2020; supervisors Igor Shparlinski and Liangyi Zhao.

(C) 2020 Australian Mathematical Publishing Association Inc. 
proof technique uses the Harman sieve [6] together with the arithmetic information supplied by bounds for multiplicative character sums.

In Chapter 3, we consider yet another variant of EOSC. We obtain an asymptotic formula for the number of ways to represent every reduced residue class as a product of a prime and a square-free integer. Specifically, for $(a, q)=1$, we count the number of pairs $(p, s)$ such that

$$
p s \equiv a(\bmod q),
$$

where prime $p \leq P$, square-free $s \leq S$. The main tool is a bound for the Kloostermann sum over primes provided by Fouvry and Shparlinski [5].

In Chapter 4 , we derive new lower bounds on the number of smooth square-free integers up to $x$ in residue classes modulo a prime $p$, relatively large compared to $x$, which in some ranges of $p$ and $x$ improve that of Balog and Pomerance [1]. We also obtain an estimate on the smallest square-full integer in almost all residue classes modulo a prime $p$. This is joint work with Munsch and Shparlinski and has been published in [9].

In Chapter 5, we consider a relaxation of the binary Goldbach conjecture, which states that all even integers greater than two can be written as the sum of two primes. Uniformly for small $q$ and $(a, q)=1$, we obtain an estimate for the weighted number of ways a sufficiently large integer $N$ can be represented as the sum of a prime congruent to $a$ modulo $q$ and a square-free integer. That is, we obtain an estimate for the quantity

$$
\mathcal{R}_{a, q}(N):=\sum_{\substack{N=p+n \\ p \equiv a(\bmod q)}} \mu^{2}(n) \log p .
$$

Here $\mu$ is the Möbius function. Our method is based on the notion of a local model developed by Ramaré [10] and may be viewed as an abstract circle method.

In Chapter 6, we provide a refinement of the classical Burgess bound for multiplicative character sums modulo a prime number $q$. This continues a series of previous logarithmic improvements, which are mostly due to Iwaniec and Kowalski [7]. In particular, for any nontrivial multiplicative character $\chi$ modulo a prime $q$ and any integer $r \geq 2$, we show that

$$
\sum_{M<n \leq M+N} \chi(n)=O\left(N^{1-1 / r} q^{(r+1) / 4 r^{2}}(\log q)^{1 / 4 r}\right),
$$

which sharpens previous results by a factor of $(\log q)^{1 / 4 r}$. Our improvement comes from averaging over numbers with no small prime factors rather than an interval as in previous approaches. This is joint work with Kerr and Shparlinski [8]. Finally, we remark that de la Bretèche and Munsch [2] and de la Bretèche et al. [3] have improved our result building on this idea. 


\section{References}

[1] A. Balog and C. Pomerance, 'The distribution of smooth numbers in arithmetic progressions', Proc. Amer. Math. Soc. 115(1) (1992), 33-43.

[2] R. de la Bretèche and M. Munsch, 'Minimizing GCD sums and applications to non-vanishing of theta functions and to Burgess' inequality', Preprint, 2019, arXiv:1812.03788.

[3] R. de la Bretèche, M. Munsch and G. Tenenbaum, 'Small Gál sums and applications', J. London Math. Soc., to appear.

[4] P. Erdős, A. M. Odlyzko and A. Sárközy, 'On the residues of products of prime numbers', Period. Math. Hungar. 18(3) (1987), 229-239.

[5] E. Fouvry and I. E. Shparlinski, 'On a ternary quadratic form over primes', Acta Arith. 150(3) (2011), 285-314.

[6] G. Harman, Prime-Detecting Sieves, London Mathematical Society Monograph Series, 33 (Princeton University Press, Princeton, NJ, 2007).

[7] H. Iwaniec and E. Kowalski, Analytic Number Theory, American Mathematical Society Colloquium Publications, 53 (American Mathematical Society, Providence, RI, 2004).

[8] B. Kerr, I. E. Shparlinski and K. H. Yau, 'A refinement of the Burgess bound for character sums, Michigan Math. J. 69(2) (2020), 227-240.

[9] M. Munsch, I. E. Shparlinski and K. H. Yau, 'Smooth squarefree and squarefull integers in arithmetic progressions', Mathematika 66(1) (2020), 56-70.

[10] O. Ramaré, Arithmetical Aspects of the Large Sieve Inequality, Harish-Chandra Research Institute Lecture Notes, 1 (Hindustan Book Agency, New Delhi, 2009).

[11] K. H. Yau, 'Distribution of $\alpha n+\beta$ modulo 1 over integers free from large and small primes', Acta Arith. 189(1) (2019), 95-107.

KAM HUNG YAU, School of Mathematics and Statistics,

University of New South Wales, Kensington, New South Wales, 2052,

Australia

e-mail: z5128837@unsw.edu.au 